\title{
CONSPECTVS SIGLORVM
}

\section{CODICES PHAEDRI FABVLARVM}

P codex Pithoeanus (New York, Pierpont Morgan Library, M. 906, ff. 33-87), saec. $\mathrm{IX}^{3 / 4-4 / 4}$ conscriptus, membranaceus. Ff. 33-71, pluribus manibus exaratis, continentur Phaedri fabulae.

R codex Remensis, saec. $\mathrm{IX}^{2 / 4}$ una manu exaratus, membranaceus, anno 1774 combustus. Textus codicis $\mathbf{R}$ cognoscitur per haec testimonia (uide Praef. $\S$ II):

$\mathbf{R}^{\mathbf{R}}$ testimonia Rigaltii in Phaedri fabularum editionibus annorum 1617 et 1630 .

$\mathbf{R}^{\mathbf{s}} \quad$ lectiones quas Jacobus Sirmond ex $\mathbf{R}$ deprompsit et in unum folium chartaceum congessit, quod asseruatur Parisiis in Bibliotheca nationali (Baluze 141, f. 211r-u).

$\mathbf{R}^{\text {Sir }}$ coniecturae quas Sirmondus in folio chartaceo nunc nuper laudato interiecit lectionibus codicis $\mathbf{R}$. Coniecturis suis Sirmondus praeposuit compendium 'F.' (id est 'Fortasse'), lectionibus autem codicis $\mathbf{R}$ compendium ' $\mathrm{V}$.' (id est 'Vetus codex').

$\mathbf{R}^{\mathbf{g}}$ lectiones quas $\delta \iota \alpha \rho \eta \dot{\delta} \delta \eta$ codici $\mathbf{R}$ tribuit Marquardus Gude in adnotationibus ad Phaedrum publicatis in Petri Burmanni maioris editione Phaedri fabularum typis expressa anno 1698.

$\mathbf{R}^{\text {? }}$ lectiones quae in Gudii adnotationibus a Burmanno 1698 uulgatis notantur compendio "MS". Quae lectiones a Gudio haustae esse possunt aut ex $\mathbf{R}$ aut ex $\mathbf{P}$.

$\mathbf{R}^{\mathbf{r}}$ testimonia Dionysii Roche, qui anno 1665 haud pauca ex $\mathbf{R}$ exscripsit in schidis quae nunc asseruantur Parisiis in Bibliotheca Sorbonae (MS 1170, ff. 3 sqq.).

$\mathbf{R}^{\mathbf{v}}$ lectiones quas $\delta \iota \alpha \rho \rho \eta \dot{\delta} \eta \nu$ codici $\mathbf{R}$ attribuit Jacobus Claudius Vincent. Hic uir doctus instituit collationem codicis $\mathbf{R}$ et Phaedri fabularum editionis quae Parisiis apud uiduam Brocas anno 1743 prodierat. Vincentii collationem publici iuris fecit Berger de Xivrey 1830. Vide etiam quae infra ad siglum $\mathbf{r}$ explicandum scribam. 
$\rho$ editio Parisiensis apud uiduam Brocas anno 1743 uulgata, quacum Jacobus $\mathrm{Cl}$. Vincent, ut supra dixi, contendit codicem R. Siglum $\boldsymbol{\rho}$ apposui lectionibus Brocasianae editionis quae e Vincentii silentio codici $\mathbf{R}$ assignari possunt.

$\Delta$ lectiones editionis Phaedri fabularum a Petro Danet anno 1726 in lucem emissae quae e Jacobi Cl. Vincent silentio codici $\mathbf{R}$ attribui possunt. Nam ab editione apud uiduam Brocas impressa ( $\rho$ ), qua Vincentius usus est in collatione facienda, nonnullae fabulae absunt; harum igitur textum quem $\mathbf{R}$ exhibebat idem Vincentius cum Daneti editione comparauit.

$\mathbf{R}^{\mathbf{b}}$ testimonia Gabrielis Brotier in Phaedri editione anni 1783 (cf. Praef. adn. 190).

$\mathbf{R}^{\mathbf{p}}$ fabulae de uulpe ad personam tragicam (Phaedr. 1, 7) imago a Le Vacher, bibliothecario Remensi, delineata et cum N.-A. Pluche communicata; quam imaginem ipse Pluche aeri incidendam curauit et anno 1746 edidit.

$\mathbf{R}^{\mathbf{f}} \quad$ specimina quae ex $\mathbf{R}$ anno 1769 Jacobus $\mathrm{Cl}$. Vincent palaeographica fide descripsit charta pellucida usus.

$\mathbf{r}$ correctiones codicis $\mathbf{R}$ uetustae, per testimonia Vincentii a Bergero de Xivrey edita notae. Quotienscumque hae correctiones in apparatu critico laudantur, totiens siglum $\mathbf{R}^{\mathbf{v}}$ idem significat quod ' $\mathbf{R}$ ante correctionem teste Vincentio'.

D codex Reginensis Latinus 1616, qui in bibliotheca Vaticana asseruatur, membranaceus. Ff. $17 \mathrm{r}-18 \mathrm{r}$, saec. $\mathrm{IX}^{2 / 3}$ exaratis, continentur octo fabulae libri primi Phaedri fabularum (11, $12,13,17,18,19,20,21)$.

N codex Neapolitanus siue Perottinus (Napoli, Biblioteca Nazionale, IV F 58), testis unicus Nicolai Perotti 'Epitomes fabellarum Aesopi Auieni et Phaedri', chartaceus, post a. 1474, ut uidetur, exaratus. Codex autographus est. Insunt 63 Phaedri carmina, poematis aliquot Auiani et Perotti ipsius intermixta. $\mathbf{N}$ ita madore ac situ corruptus est ut locis permultis ne Lyncea quidem oculorum acie legi queat. Lacunae tamen 
expleri possunt ope duorum codicum ex $\mathbf{N}$ nondum pessumdato descriptorum:

V hac littera notatur codex Vrbinas Latinus 368, membranaceus, circa a. 1482 a Federico Veterano exaratus, qui continet ff. 100-146 Perottinam fabellarum epitomen totam et integram.

U codex Vrbinas Latinus 301, chartaceus, inter annos 1477 et 1480 exaratus, testis unicus Perotti 'Cornus copiae'. Hic codex non est autographus; Perottus tamen sua ipsius manu multa addidit, multa correxit. Continet duas Phaedri fabulas, a calligrapho exscriptas, 3,17 f. 644 r, app. 4 f. $126 \mathrm{r}-\mathrm{u}$.

Vbi scriptura codicis $\mathbf{N}$ euanuit respicienda sunt etiam quae de eo priores rettulerunt. Quapropter in meo apparatu critico citantur:

$\mathbf{N}^{\mathbf{0}}$ sylloges fabellarum Perottinae exemplar quod ex $\mathbf{N}$ Jacobus Philippus D’Orville anno 1727 sua ipsius manu descripsit; quod exemplar nunc Oxonii in bibliotheca Bodleiana asseruatur (ms. D'Orville 524).

$\mathbf{N}^{\mathbf{b}}$ codicis $\mathbf{N}$ lectiones publici iuris factae a Petro Burmanno maiore in praefatione ad Phaedri fabularum editionem quae Leidae anno 1727 prodiit ipso curante; quas lectiones ad Burmannum transmiserat Dorvillius.

$\mathbf{N}^{\mathbf{i}}$ codicis $\mathbf{N}$ editio a Cataldo Iannelli curata (= Iannelli 1809).

$\mathbf{N}^{\mathbf{d}}$ codicis $\mathbf{N}$ lectiones a Dominico Bassi allatae in Phaedri fabularum editione anno 1918 impressa.

n uide, quaeso, ad 3, 13.

M codex Vaticanus Latinus 5190, chartaceus. Ff. 111r-125r, saec. $\mathrm{xv}^{2}$ duabus manibus exaratis, continentur 22 Phaedri fabulae, quae carminibus aliquot Auiani interponuntur. Altera manus, prioris fere aequalis, f. $125 \mathrm{r}$ addidit Phaedr. 3,9 et 3, 10, 1-16. 


\section{TESTES PARAPHRASIS T}

ПI Р. Oxy. 1404, saec. III exarata.

$Y^{\mathbf{L}}$ pars fabularum 'Pseudodositheanarum' codice Leidensi (Leiden, Bibliotheek der Rijksuniversiteit) Voss. Gr. Q. 7, membranaceo, saec. $\mathrm{IX}^{2 / 4}$ conscripto, traditarum quae ex $\mathbf{T}$ pendet.

$\mathbf{Y}^{\mathbf{P}}$ pars fabularum 'Pseudodositheanarum' codice Parisiensi (Paris, Bibliothèque nationale de France) Lat. 6503, membranaceo, saec. $\mathrm{ix}^{3 / 4}$ exarato, traditarum quae ex $\mathbf{T}$ pendet.

B codex Londiniensis (London, British Library) Burneianus 59, saec. $\mathrm{XI}^{1 / 4}$ exaratus, membranaceus.

Ad codex Leidensis (Leiden, Bibliotheek der Rijksuniversiteit) Voss. Lat. O. 15, membranaceus, ab Ademaro Cabannensi ( $†$ 1034) exaratus. Insunt fabulae quae de $\mathbf{T}$ per $\chi$ huc fluxerunt, fabulae quae ex $\mathbf{P h}$ pendent. Fabulae quae ex $\boldsymbol{\chi}$ deriuatae sunt siglo $\mathbf{A d} \chi$ a me designantur, ceterae $\mathbf{A d}^{\mathbf{P h}}$. Fabellae aliquot textum contaminatum praebent: has $\mathbf{A d}^{\mathbf{P h} \chi}$ denoto si textum paraphrasis $\mathbf{P h}$ interpolatum ex lectionibus codicis $\chi$ exhibent; $\mathbf{A d} \mathbf{\chi}^{\mathbf{P h}}$ si textum codicis $\chi$ contaminatum lectionibus paraphrasis $\mathbf{P h}$.

F codex Laurentianus (Firenze, Biblioteca Medicea Laurenziana) Ashburnhamianus 1555, membranaceus, saec. XIII ${ }^{1}$ conscriptus.

Mn codex Cenomanensis (Le Mans, Médiathèque Louis Aragon) 84 , membranaceus, saec. XIII $^{2}$ conscriptus.

S hoc siglo insigniuntur fabulae soluta oratione conscriptae quas Henricus Steinhöwel ex nescioquo codice nunc deperdito desumpsit et in 'Esopo' suo circa a. 1476-1477 edidit.

Wi codex Vindobonensis (Wien, Österreichische Nationalbibliothek) 901, membranaceus, saec. XIII ${ }^{2}$ exaratus. Aliae fabulae Aesopiae prosa oratione conscriptae quae hoc codice continentur textum codicis $\boldsymbol{\sigma}$ exhibent $\left(=\mathbf{W i}^{\boldsymbol{\sigma}}\right)$, aliae textum codicis $\tau\left(=\mathbf{W i}^{\tau}\right)$, reliquae textum contaminatum: has siglo 
$\mathbf{W} \mathbf{i}^{\boldsymbol{\sigma} \tau}$ insigniui si fons primarius est $\boldsymbol{\sigma}$; sin autem fons primarius est $\tau$, siglo $\mathbf{W} \mathbf{i}^{\tau \sigma}$.

Fr codex Francofurtensis (Frankfurt am Main, Universitätsbibliothek) Praed. 60, chartaceus, inter annos 1442 et 1444 a J. Streler conscriptus. Insunt fabulae Aesopiae, anno 1443 exaratae, quarum pars ex $\chi$ pendet $(=\mathbf{F r} \chi)$, pars ex $\mathbf{l}\left(=\mathbf{F r}^{\mathfrak{l}}\right)$.

H codex Guelf. (Wolfenbüttel, Herzog August Bibliothek) 699 Helmst., chartaceus, saec. $\mathrm{xv}^{1 / 3}$ exaratus. Siglo $\mathbf{H}^{v}$ notantur fabulae quae ex $\boldsymbol{v}$ deriuatae sunt.

E codex Berolinensis (Berlin, Staatsbibliothek, Preussischer Kulturbesitz) Lat. O. 87, saec. $\mathrm{XII}^{2}$ exaratus, membranaceus.

Vi codex Vindobonensis (Wien, Österreichische Nationalbibliothek) 303 , membranaceus, saec. XIII ${ }^{3 / 4}$ exaratus.

Mk codex Mellicensis (Melk, Stiftsbibliothek) 1916, saec. $\mathrm{xv}^{3 / 4}$ conscriptus, chartaceus.

L Anonymi 'Liber de moribus egregiisque dictis omnium philosophorum et poetarum' (saec. XIV ${ }^{\text {med}}$ ), ubi capiti de uita Aesopi subiunguntur fabulae prosariae ex $\mathbf{o}$ ascitae. In $\mathbf{L}$ textus codicis o saepissime contaminatus est textu cuiusdam codicis Gualterii Anglici fabularum, interdum lectionibus nesciocuius libri manu scripti Phaedri carminum nunc deperditi $(=\zeta)$. Hos codices nondum typis excusi 'Libri de moribus' contuli:

$\mathbf{L}^{\mathbf{a}}$ codicem Monacensem (München, Bayerische Staatsbibliothek) Clm 26781, chartaceum, anno 1441 exaratum,

$\mathbf{L}^{\mathbf{b}}$ codicem Monacensem (München, Bayerische Staatsbibliothek) Clm 14129, chartaceum, circa a. 1450 conscriptum.

W codex Guelf. (Wolfenbüttel, Herzog August Bibliothek) Gud. Lat. 148 , membranaceus, saec. $\mathrm{IX}^{4 / 4}$ exaratus. 


\section{TESTES PARAPHRASIS Ph}

Ad $^{\text {Ph }}$ testis fere unicus; uide quae supra ad siglum Ad explicandum scripsi.

$\mathbf{H}^{\Phi}$ in una fabula accedit codex $\mathbf{H}$ (de quo uide supra), nam $\lambda$ óyos de uulpe et aquila (cf. Phaedr. 1, 28) in $\mathbf{H}$ ex $\mathbf{P h}$ et $\boldsymbol{\chi}$ per $\boldsymbol{\Phi}$ defluxit; in altera fabula (cf. Phaedr. 1, 8) $\mathbf{H}$ praebet textum codicis $\boldsymbol{v}$ contaminatum paraphrasi $\mathbf{P h}\left(=\mathbf{H}^{\mathbf{v \Phi})}\right.$; cf. Praef. § V.

\section{LIBRI MANV SCRIPTI DEPERDITI}

$\boldsymbol{\delta}$ fons codicum $\mathbf{P} \mathbf{R}$

$\boldsymbol{\theta}$ fons codicis $\boldsymbol{\delta}$

$v$ fons $\mathbf{D} \mathbf{P h}$

$\lambda$ consensus codicum $\mathbf{N} \mathbf{M}$; hoc siglo utor si $\mathbf{N}$ legi potest et cum $\mathbf{M}$ conspirat

$\boldsymbol{\mu}$ antigraphum codicis $\mathbf{M}$

$\mathbf{Y}$ fons $\mathbf{Y}^{\mathrm{L}} \mathbf{Y}^{\mathrm{P}}$

v fons $\mathrm{B} \mathrm{Mn} \mathbf{H}^{v}$

$\boldsymbol{\sigma}$ fons $\boldsymbol{v} \mathbf{F} \mathbf{W i}^{\boldsymbol{\sigma}}$

$\xi$ fons $\boldsymbol{\sigma} \mathbf{S}$

$\chi \quad$ fons $\xi \operatorname{Ad} x$ Fr $\chi$

$\eta \quad$ fons Vi Mk

o fons $\mathbf{L}^{\mathbf{a b}} \boldsymbol{\eta}$

$\tau$ fons $\mathbf{W} \mathbf{i}^{\tau} \mathbf{E}$

$\varepsilon$ fons $0 \tau$

$\kappa$ fons $\chi \varepsilon$

I fons W $\mathbf{F r}^{\mathbf{l}}$ 
$\mathbf{T}$ fons $\boldsymbol{\kappa} \mathbf{\mathbf { ~ }}(\mathbf{W})$; accedit interdum $\mathbf{Y}$, et in fabula de cane et carne (cf. Phaedr. 1, 4) $\Pi$

$\alpha \quad$ fons $v \lambda \mathrm{T}$

$\boldsymbol{\varphi}$ fons $\boldsymbol{\theta} \boldsymbol{\alpha}$, id est liber archetypus

$\zeta \quad$ uide quae ad $\mathbf{L}$ adnotaui

Ф cf. Praef. § V

SIGLA CETERA
$\mathbf{R}^{\text {sgrv }} \quad \mathbf{R}^{s} \mathbf{R}^{\mathrm{g}} \mathbf{R}^{\mathrm{r}} \mathbf{R}^{\mathbf{v}}$
Nobid $\quad \mathbf{N}^{o} \mathbf{N}^{b} \mathbf{N}^{i} \mathbf{N}^{d}$
Pc hoc et similibus siglis correctiones denotantur librarii ipsius uel manuum librario fere aequalium
$\mathbf{W}^{2} \quad$ manus recentior in $\mathbf{W}$
$\langle$ aaa litterae e coniectura additae
$\langle * *\rangle \quad$ lacuna unius uersus
***** quot uersus exciderint incertum
[aaa litterae delendae
(aaa) litterae per compendium expressae
【aaa】 litterae a librario aut correctore deletae
'aaa' litterae a librario se ipso corrigente insertae aut additae a diorthota
$\{$ textus hinc periit
\} hinc codex denuo legi potest
$\{\ldots\}$ tres litterae deperditae
... trium litterarum uestigia dubia
ạạ tres litterae incertae sed non prorsus dubiae 
Łaaa litterae deperditae sed ex alio testimonio redintegratae

| $\quad$ finis lineae in codice

/ finis uersus

... unum pluraue uerba breuitatis causa consulto praetermisi

f. $1 \mathrm{r}^{\mathrm{a}} \quad$ columna prior folii 1 recti (uel, si mauis, aduersi)

f. $1 \mathrm{u}^{\mathrm{b}} \quad$ columna altera folii 1 uersi (uel, si mauis, auersi)

1. red. litteris redintegratis

u. 1. uaria lectio

Quod ad compendia attinet quibus in locis similibus colligendis utor cf. Praef. § XII. De nominibus operibusque uirorum doctorum uide Praef. adn.* et ea quae Indici philologorum praemisi. 
